\title{
A EXTENSÃO UNIVERSITÁRIA E A VIVÊNCIA DA PEDAGOGIA SOCIAL: A EXPERIÊNCIA DO CENTRO DE FORMAÇÃO DE PROFESSORES EM CAJAZEIRAS-PB
}

\author{
Maria Gerlaine Belchior Amaral ${ }^{1}$ \\ ORCID: 0000-0002-0745-7594 \\ Aparecida Carneiro Pires ${ }^{2}$ \\ ORCID: 0000-0001-6219-585X \\ Maria de Lourdes Campos 3 \\ ORCID: 0000-0002-1237-7863 \\ Raimunda de Fátima Neves CoÊlho 4 \\ ORCID: 0000-0002-6495-0924
}

Resumo: O texto que segue é o registro das vivências em Pedagogia Social, por meio da extensão universitária, realizadas no ano de 2018, no curso de Pedagogia, da Unidade Acadêmica de Educação, do Centro de Formação de Professores, da Universidade Federal de Campina Grande, no Câmpus de Cajazeiras - PB. Traz o relato de experiência de um Programa de Extensão intitulado "Pedagogia Social: práticas educativas em contextos diversos" composto por seis Projetos que se realizaram em quatro municípios distintos. O referido Programa foi coordenado pela Professora Dra. Maria Gerlaine Belchior Amaral e, os Projetos foram coordenados por três docentes, a saber: Professora Dra. Maria de Lourdes

1 Dra. em Educação, profa. Adjunta, Campus de Cajazeiras pela Universidade Federal de Campina Grande - UFCG. E-mail: gerlaine.ufcg@yahoo.com.br

2 Dra. em Educação, profa. Adjunta, Campus de Cajazeiras pela Universidade Federal de Campina Grande - UFCG. E-mail: cidaufcg2017@gmail.com

3 Dra. em Medicina e Saúde, profa. Adjunta, Campus de Cajazeiras pela Universidade Federal de Campina Grande - UFCG. E-mail: mlcampos_10@yahoo.com.br

4 Dra. em Medicina e Saúde, profa. Associada, Campus de Cajazeiras pela Universidade Federal de Campina Grande - UFCG. E-mail: raimunda.neves6@gmail.com 
Campos, Professora Dra. Aparecida Carneiro Pires e pela Professora Dra. Raimunda de Fátima Neves Coêlho. Este Programa de extensão universitária teve o propósito de contribuir com a formação dos graduandos do curso de Pedagogia do CFP/UFCG, oportunizando uma experiência extensionista em Pedagogia Social. Objetivou também, promover a interlocução da universidade com a sociedade ao desenvolver práticas educativas em espaços diversos. A metodologia vivenciada pelo Programa, em seus 06 subprojetos foi lúdica e interativa. Buscouse por meio de diferentes linguagens construir aprendizagens, a partir de um processo críticoreflexivo, na perspectiva de aperfeiçoar a formação dos (as) graduandos (as) de Pedagogia através de vivências de práticas interdisciplinares semanais, com as crianças, abordando as diferentes áreas do conhecimento: Matemática, Língua Portuguesa, História e Geografia e Ciências. Vivenciada de forma lúdica por meio de leituras, contação de história, produção textual, desenhos, jogos educativos, dinâmicas, músicas, entre outras. Com relação aos valores espirituais foram trabalhados por meio de exposições e discussões que suscitassem o respeito, justiça, a solidariedade, sobretudo, o amor ao próximo, propondo sempre uma interlocução com um ensino ético. Quanto aos resultados é possível concluir que nos diferentes espaços onde se efetivou oportunizou vivências que promovessem o desenvolvimento humano, cognitivo, emocional e espiritual das pessoas atendidas e, desse modo, configurou-se como um instrumento de exercício de cidadania para mulheres, crianças e adolescentes que, de certo modo, encontravam-se em situação de vulnerabilidade social. Nos tempos hodiernos, a Pedagogia Social precisa assumir um compromisso ético de formar para a cidadania, para as relações sociais, para a vida em seu aspecto político, moral e crítico.Palavras-chave: Educação em saúde. Redes sociais. Saúde da comunidade. Tecnologia da informação.

Palavras-chave: Extensão Universitária. Pedagogia Social. Vulnerabilidade Social. Formação Docente.

\title{
UNIVERSITY EXTENSION PROGRAMS AND THE DAILY PRACTICE OF SOCIAL PEDAGOGY: THE EXPERIENCE OF THE TEACHER TRAINING CENTER IN CAJAZEIRAS-PB
}

\begin{abstract}
The following work is the record of daily practices in Social Pedagogy as they took place through university extension programs, developed in 2018, in the Pedagogy Course of the Academic Unit of Education of the Teacher Training Center from the Federal University of Campina Grande in the Campus of Cajazeiras of the state of Paraíba. It deals with the report on an Extension Program titled "Social Pedagogy: educational practices in diverse contexts" composed of six Projects that took place in four different municipalities. The Program was coordinated by Professor Maria Gerlaine Belchior Amaral and the Projects were carried out by three professors: Professor Maria de Lourdes Campos, Professor Aparecida Carneiro Pires and Professor Raimunda de Fátima Neves Coêlho. This university extension program aimed at contributing to the training of undergraduates of the Pedagogy course of CFP/UFCG, by offering a practice on university extension in Social Pedagogy. It also aimed at promoting the interlocution of the university with society by developing educational practices in different spaces. The methodology used by the Program in its six subprojects was of a playful and
\end{abstract}


interactive nature. It was sought through different approaches to promote learning, based on a critical-reflexive process, with the perspective of improving the training of Pedagogy undergraduates through experiences of weekly interdisciplinary practices with children, addressing different areas of knowledge such as Mathematics, Portuguese Language, History, Geography and Sciences. Every activity was carried out in a playful way through readings, storytelling, textual production, drawings, educational games, dynamics and musical exercises, among other practices. With regard to human values, they were worked through expositions and discussions focusing on major concepts of respect, justice, solidarity and, above all, love of humanity, consistently proposing a dialogue that would lead to an ethical lesson. As for results, it is possible to conclude that in the different spaces where the program took place, it helped to establish daily practices where human, cognitive, emotional and spiritual values became important tools for the assisted communities in exercising their citizenship, especially for women, children and adolescents who, in a sense, were in a situation of social vulnerability. In modern times, Social Pedagogy is called to come up to the challenge of enforcing an ethical commitment to encourage citizenship and social relations based on moral, political, and critical aspects of life.

Keywords: University. Extension. Social Pedagogy. Social Vulnerability. Teacher Training.

\section{LA EXTENSIÓN UNIVERSITARIA Y LA VIVENCIA DE LA PEDAGOGÍA SOCIAL: LA EXPERIENCIA DEL CENTRO DE FORMACIÓN DE PROFESORES EN CAJAZEIRAS-PB}

Resumen: El texto que sigue es el registro de las vivencias en Pedagogía Social, por medio de la extensión universitaria, realizadas en el año 2018, en el curso de Pedagogía, de la Unidad Académica de Educación, del Centro de Formación de Profesores, de la Universidad Federal de Campina Grande, en el Cámpus de Cajazeiras - PB. Trae el relato de experiencia de un Programa de Extensión titulado "Pedagogía Social: prácticas educativas en contextos diversos" compuesto por seis Proyectos que se realizaron en cuatro municipios distintos. El programa fue coordinado por la Profesora Dra. Maria Gerlaine Belchior Amaral y, los Proyectos fueron coordinados por tres docentes, a saber: Profesora Dra. Maria de Lourdes Campos, Profesora Dra. Aparecida Carneiro Pires y por la Profesora Dra. Raimunda de Fátima Neves Coêlho. Este programa de extensión universitaria tuvo el propósito de contribuir con la formación de los graduandos del curso de Pedagogía del CFP / UFCG, oportunizando una experiencia extensionista en Pedagogía Social. Objetivó también, promover la interlocución de la universidad con la sociedad al desarrollar prácticas educativas en espacios diversos. La metodología vivenciada por el Programa, en sus 06 subproyectos fue lúdica e interactiva. Se buscó por medio de diferentes lenguajes construir aprendizajes, a partir de un proceso crítico-reflexivo, en la perspectiva de perfeccionar la formación de los graduados de Pedagogía a través de vivencias de prácticas interdisciplinares semanales, con los niños, abordando las diferentes áreas de conocimiento: Matemáticas, Inglés Lengua, Historia y Geografía y Ciencia. Vivenciada de forma lúdica por medio de lecturas, narración de historia, producción textual, dibujos, juegos educativos, dinámicas, músicas, entre otras. Con respecto 
a los valores espirituales fueron trabajados por medio de exposiciones y discusiones que suscitar el respeto, la justicia, la solidaridad, sobre todo, el amor al prójimo, proponiendo siempre una interlocución con una enseñanza ética. En cuanto a los resultados es posible concluir que en los diferentes espacios donde se efectuó oportunizó vivencias que promovían el desarrollo humano, cognitivo, emocional y espiritual de las personas atendidas y, de ese modo, se configuró como un instrumento de ejercicio de ciudadanía para mujeres, niños y adolescentes que, en cierto modo, se encontraban en situación de vulnerabilidad social. En los tiempos actuales, la Pedagogía Social necesita asumir un compromiso ético de formar para la ciudadanía, para las relaciones sociales, para la vida en su aspecto político, moral y crítico.

Palabras clave: Extensión Universitaria. Pedagogía Social. Vulnerabilidad Social. Formación Docente.

\section{CONSIDERAÇÕES INICIAIS}

Este Programa de extensão universitária intitulado "Pedagogia Social: práticas educativas em contextos diversos" teve a finalidade de contribuir com a formação dos graduandos do curso de Pedagogia, da Unidade Acadêmica de Educação (UAE), do Centro de Formação de Professores da Universidade Federal de Campina Grande-CFP/UFCG, oportunizou uma experiência extensionista em Pedagogia Social. Tendo como abordagem um segmento da Pedagogia que se efetiva em contextos informais, tais como: igrejas, sindicatos, Organizações Não Governamentais- ONGs, Casa de Acolhimento, Centros Comunitários, residência dos próprios estudantes, entre outros. Então, a Pedagogia Social está em consonância com a Resolução CNE/CP nº. 01, de 15 de maio de 2006 que institui as Diretrizes Curriculares Nacionais para o Curso de Graduação em Pedagogia, que no Art. 5, Inci $\neg$ so IV, estabelece que o egresso do curso de Pedagogia deverá estar apto a "trabalhar, em espaços escolares e não $\neg$-escolares, na promoção da aprendizagem de sujeitos em diferentes fases do desenvolvimento humano, em diversos níveis e modalidades do processo educativo". (BRASIL, 2006).

O Programa aqui relatado promoveu ações educativas para crianças, adolescentes e mulheres em situação de vulnerabilidade social. Foi desenvolvido por meio de 06 subprojetos que foram implementados nas cidades de Cajazeiras-PB, Sousa-PB, Santa Helena-PB e no distrito de Felizardo-CE no período compreendido de maio a dezembro de 2018, sob coordenação geral da Profa. Dra. Maria Gerlaine Belchior Amaral. Segue a descrição dos projetos que integravam o Programa: 
O projeto 01- Pedagogia Social: a interlocução entre espiritualidade e educação sob a coordenação da Profa. Dra. Aparecida Carneiro Pires foi desenvolvido na igreja Nossa Senhora da Conceição, no distrito de Felizardo-CE. Objetivou compreender os fundamentos teóricos e práticos da Pedagogia Social; vivenciar ações que promovam a socialização e potencialize o desenvolvimento cognitivo e, também desenvolver práticas educativas que contribuam para reflexão e consolidação dos valores humanos, tais como: solidariedade, respeito, cuidado com o outro, generosidade, justiça, diálogo, entre outros.

O projeto 02: Pedagogia Social: a contribuição do pedagogo ao desenvolvimento cognitivo dos residentes no Centro de atenção à Criança e ao Adolescente (CCA) em Cajazeiras-PB sob a coordenação da Profa. Dra. Maria de Lourdes Campos aconteceu na Casa de Acolhimento às Crianças que estão sob Medida Protetiva, na cidade de Cajazeiras-PB. Objetivou compreender os fundamentos teóricos e práticos da Pedagogia Social; vivenciar ações que promovam a socialização e potencialize o desenvolvimento, motor, emocional, cognitivo e o raciocínio logico matemático das crianças e adolescentes residentes no CCA e, ainda, desenvolver práticas educativas que contribuam para reflexão e consolidação dos valores humanos, tais como: solidariedade, respeito, cuidado com o outro, generosidade, justiça, diálogo, entre outros.

O projeto 03- Pedagogia Social: a interlocução entre a música e a educação sob a coordenação da Profa. Dra. Maria Gerlaine Belchior Amaral na própria residência de uma graduanda do curso de Pedagogia - prática que se efetivou durante todo o ano de 2018 e objetivou compreender os fundamentos teóricos e práticos da Pedagogia Social - Ampliar os conhecimentos músico-vocais; favorecer o desenvolvimento cognitivo a partir da musicalidade e, ainda, proporcionar uma formação humana agregada a princípios éticos de respeito, justiça e solidariedade para com o próximo.

O projeto 4 - Pedagogia Social: educação e valores humanos sob a coordenação da Profa. Dra. Aparecida Carneiro Pires desenvolveu-se na Igreja Matriz de Santa Helena. Objetivou estimular as habilidades de leitura e interpretação de textos e a resolução de problemas com a utilização de jogos e brincadeiras; despertar nas crianças o gosto pela leitura e escrita com vários gêneros textuais; trabalhar a Matemática com ludicidade; usar a arte como auxílio para o desenvolvimento cognitivo das crianças nessas disciplinas; valorizar o conhecimento próprio da criança, a fim de expandi-lo, além de procurar desenvolver a criança em atividades que promovam seu bem-estar físico e social. 
O projeto 05 - Práticas socioeducativas com crianças e adolescentes residentes no Centro de Acolhimento Credente Vides em Sousa-PB. Coordenadora: Profa. Dra. Maria de Lourdes Campos foi desenvolvido com residentes do Centro de Acolhimento Credente Vides, na cidade de Sousa-PB. Objetivou compreender os fundamentos teóricos e práticos da Pedagogia Social; desenvolver atividades que promovam uma relação fundamentada nos valores éticos e morais das crianças residentes do Centro de Acolhimento Credente Vides; promover a diversidade cultural e direitos humanos através do lúdico e, também contribuir com o desenvolvimento cognitivo e humano das crianças.

O projeto 06 - Passageiras vítimas da violência: de seus espaços diversos para o Centro de Referência de Assistência à Mulher (CRAM Susane Alves em Cajazeiras-PB. Coordenadora: Profa. Dra. Raimunda de Fátima Neves Coêlho foi desenvolvido no Centro de Referência de Assistência à Mulher (CRAM). Objetivou fomentar práticas educativas com mulheres vítimas da violência, de modo a intervir a partir de suas singularidades; caracterizar o perfil das mulheres violentadas; vivenciar oficinas psicopedagógicas para superação de seus conflitos pessoais e coletivos, de modo a humanizar-se; discutir itinerários humanos-inumanos, vivências injustas, porém, resistentes de sujeitos coletivos - as mulheres e ainda, ressignificar as formas de resistência no convívio com esse coletivo, tendo em vista a superação do medo, na perspectiva de anúncio e denúncia.

As ações desenvolvidas por este Programa tiveram aporte teórico nos fundamentos teóricos da pedagogia freireana e da Pedagogia Social, a partir da qual a educação é entendida como instrumento político, uma prática de liberdade e, acima de tudo, um instrumento eficaz de transformação social; bem como promover uma interlocução da universidade com a sociedade por meio da vivência de práticas socioeducativas em espaços diversos atendendo grupos sociais que de algum modo encontram-se em situação de vulnerabilidade social.

$\mathrm{Na}$ acepção de Graciani (2014, p. 21)

A Pedagogia Social suscita a reflexão e ação junto aos excluídos, respeitando e validando suas histórias pessoais de subsistência e sobrevivência como representações que denunciam a crueldade da realidade social, tendo em vista a sua promoção político-social.

Ampliando esse olhar, Severo (2017) assinala que em nosso País, a Pedagogia Social se encaminha de modo a traduzir-se como um campo teórico-metodológico que abarca concepções e práticas pedagógicas voltadas especialmente para 
os âmbitos de educação não escolar, a partir de esforços sistemáticos de produção científica, formação de pesquisadores e agentes educativos em interlocução com atores e instituições do campo social, diferentes enfoques que, historicamente, têm sido representados por ideias, conceitos e termos desarticulados (SEVERO; MACHADO; RODRIGUES, 2014).

Nessa perspectiva, para o referido autor a Pedagogia Social colabora no aprofundamento da qualidade da relação entre teoria e prática na ação socioeducativa em diferentes contextos, especialmente em espaços não escolares, integrando diferentes experiências e saberes culturais que dialoguem com as formas de sociabilidades contemporâneas e contribua com a formação de valores, atitudes e condutas necessárias ao desenvolvimento humano e cultural da sociedade.

Sendo assim, Severo (2017) reitera que, a Pedagogia Social se atrela a uma concepção mais ampla de formação humana, e atualmente, reconhece-se esta área como uma disciplina científica que, assim como a Pedagogia, detém um caráter teórico-prático fundado na interface descritivo-normativa, que se ocupa da dimensão socializadora da educação em contextos sociais diversos com vistas a potencializar qualidade de vida e desenvolvimento sociocultural em uma perspectiva especial e aplicada.

Partindo destes pressupostos, é que a metodologia desenvolvida no Programa contemplou ações lúdicas, interativas e reflexivas, por meio de diferentes linguagens construir aprendizagens, a partir de um processo crítico-reflexivo, na perspectiva de aperfeiçoar a formação dos (as) graduandos (as) de Pedagogia através de vivências de práticas interdisciplinares semanais, com as crianças, abordando as diferentes áreas do conhecimento: Matemática, Língua Portuguesa, História e Geografia e Ciências. Vivenciada de forma lúdica por meio de leituras, contação de história, produção textual, desenhos, jogos educativos, dinâmicas, músicas, entre outras.

Cabe ressaltar que, a mediação dos (as) graduandos (as) foi de fundamental importância para o alcance dos objetivos propostos, uma vez que se aliou o planejamento, a organização e a materialização das atividades à sensibilidade das questões apresentadas e vividas pela comunidade nos diversos contextos atendidos. A formação dos estudantes de Pedagogia é um elemento determinante no êxito do Programa. Diferencia-se de um trabalho voluntariado em virtude da profissionalidade docente obtida na graduação em Pedagogia. É a mediação docente qualificada que leva até a sociedade os conhecimentos científicos inerentes ao âmbito acadêmico. 
Com relação a mediação Bulgraen (2010) elucida que a ação pedagógica no processo de ensino consiste, basicamente, na "prática social", e só pôde ser alcançada através de uma ação pedagógica mediadora e problematizadora dos conteúdos sistematizados, das vivências dos alunos e dos acontecimentos da sociedade atual.

Do ponto de vista operacional, as reuniões de estudo. orientações e planejamento aconteciam quinzenalmente na universidade com a presença dos (as) extensionistas bolsistas/ voluntários (as), colaboradoras, da coordenadora geral e de cada projeto, visando à partilha e o aprimoramento das atividades a serem desenvolvidas nos respectivos contextos mencionados. É pertinente destacar que o planejamento qualifica a ação docente, isso, nos leva a reconhecer e reafirmar que os encontros na universidade, quando realizados com seriedade e compromisso, constituem-se em fator de êxito das ações extensionistas.

Quanto à dimensão metodológica, as atividades foram sempre realizadas de forma lúdica, através de: contação de histórias, músicas, desenhos, leituras, apresentação de vídeos, produções textuais orais e escritas, jogos, danças, fábulas, rodas de conversas, oficinas de música, aulas de técnica vocal com exercícios de aquecimento vocal, diagnóstico inicial das tessituras vocais e sondagem dos conhecimentos prévios do aluno como ponto norteador para uma abordagem significativa dos conhecimentos vocais. Tais atividades mais direcionadas ao público de crianças e adolescentes atendidos pelo Programa.

As reflexões de Caliman (2010) traduzem a compreensão metodológica utilizada no Programa, em que se defendeu o desenvolvimento de atividades desvinculadas de uma socialização/adaptação do indivíduo, e sim, de infundir neles uma atitude crítica capaz de provocar mudanças e transformações na sociedade. Em outras palavras, não se tratou de promover adaptação e acomodação à sociedade, mas de promover a mudança na mesma. A relação entre coordenadores/as/, extensionistas e a comunidade defendia que não se tratava de exercer um papel de controle social sobre as crianças/ adolescentes, mas fazer com que estes fossem e se sentissem sujeitos de sua própria formação.

Com o público adulto, composto por mulheres a metodologia vivenciada foi por meio de oficinas, a saber: oficinas para os cuidados de si e do outro; de valorização da história de vida e cultura; oficinas de construção de ações versando temáticas como: Mulheres - sujeitos coletivos de direitos; Mulheres - sujeitos do direito a um conhecimento de libertação; Mulheres - sujeitos do direito à cultura e à diversidade; Mulheres - sujeitos do direito à memória, humanas memórias, 
pautando-se na educação popular, capaz de gerar a ação transformadora da sociedade, em que sua palavra chave é a esperança na transformação social através do dialógico, da Pedagogia crítica e "emancipadora".

O trabalho de extensão no CRAM, desencadeado pela Pedagogia Social, coloca para nós, pedagogos/as, a oportunidade de transformar vidas por meio da educação fora do contexto escolar. Ainda, a relevância de contribuir com essas mulheres que sofrem violências, é significativa por percebermos o quanto são renegados e negligenciados os direitos de defesa da mulher que sofre violência.

Desse modo, o projeto desenvolvido com essas mulheres, constituído com a realização de oficinas tem possibilitado um trabalho humanizante e de valorização do seu eu, a motivação para o enfrentamento de seus direitos e reconstrução de suas memórias, busca de sua identidade como maneira de empoderamento de ser mulher.

Os objetivos propostos para o trabalho com as mulheres vítimas de violência também foram atendidos. As oficinas e visitas realizadas possibilitaram ganho de confiança, de modo que falaram de suas histórias de vida, dos seus medos, angústias e superações. Isso tudo leva a um processo de libertação e de possibilidade de pensar novas perspectivas e saídas para a situação que vivenciam ou vivenciaram.

Os muitos relatos oportunizados durante os encontros coletivos permitem afirmar que as ações contribuíram para a formação dos (as) graduandos (as), pois permitiram que todos os envolvidos mantivessem contato direto com a sociedade, cabe destacar que esta é uma função precípua da extensão universitária, a interlocução do público universitário com pessoas que encontram-se fora dos muros acadêmicos, num diálogo profícuo no qual ambas as partes são beneficiadas.

Tais experiências os prepara para a construção de uma identidade docente eivada pela formação humana e profissional, tão necessárias na contemporaneidade. Em parte, também são preparados para o desafio da profissão docente que por natureza é demasiadamente complexa. É possível afirmar que as múltiplas experiências vivenciadas por meio da extensão constituíram-se numa oportunidade efetiva de articulação da relação teoria e prática, algo tão caro à formação docente.

Neste Programa, algo também merecedor de destaque foi o envolvimento comprometido das pessoas, de modo geral, nas diferentes atividades desenvolvidas. Conforme mencionado no início do texto, o Programa aconteceu em 
diferentes cidades e, o compartilhamento das atividades entre as cidades também se configurou como um elemento relevante a ser destacado. Os encontros efetivados em cada contexto possibilitaram aos estudantes de Pedagogia aprimorar suas práticas e refletir suas próprias ações.

O Plano Nacional de Educação (PNE) aponta como estratégia para garantir a qualidade do ensino superior que "no mínimo, dez por cento do total de créditos curriculares exigidos para a graduação em programas e projetos de ex ᄀtensão universitária”. As experiências vivenciadas no decurso deste Programa vêm reiterar que a participação dos graduandos em ações extensionistas é algo imprescindível para uma formação acadêmica qualificada. Todos os participantes foram unânimes em externar as múltiplas contribuições do Programa à sua formação pessoal e profissional.

\section{CONSIDERAÇÕES CONCLUSIVAS}

Esta experiência extensionista oportunizou a toda equipe envolvida no Programa conhecer na prática o que é a Pedagogia Social e, com isso, ampliar a visão sobre a profissão do/a pedagogo/a, para além de professor/a nas salas de aulas das escolas, para espaços não escolares.

A extensão universitária viabilizou a vivência com sujeitos em estado de vulnerabilidade social, os quais vivem sob medida protetiva em casas de acolhimento. Nesta direção, reiteramos o crescimento nas três dimensões: pessoal, intelectual e política para todos os envolvidos no Programa e subprojetos.

Portanto, a realização deste Programa propiciou de maneira efetiva a possibilidade da universidade cumprir o seu papel social por meio da extensão universitária, partilhando saberes, informações, conhecimentos e experiências socioeducativas.

Ao finalizar as atividades do Programa, muitas coisas podem ser ditas em a partir da experiência vivenciada. Houve de fato uma interlocução promissora da universidade com a sociedade. Os extensionistas voluntários, colaboradoras e bolsistas do projeto compartilharam concretamente seus saberes científicos, acadêmicos e pedagógicos para pessoas da sociedade que realmente necessitavam de tais saberes.

É possível assegurar, também, que houve aprimoramento da formação de todos os sujeitos envolvidos no Programa: bolsistas, colaboradoras, extensionistas voluntários(as) e coordenadoras. Os estudos, vivências, trocas de experiências, 
as escritas produzidas e os vários trabalhos científicos apresentados, tudo contribuiu para que o processo formativo fosse aprimorado.

Um ponto relevante a ser destacado foram os estudos teóricos, individuais e coletivos, que possibilitaram aprofundamento acerca dos fundamentos teóricos, metodológicos e legais que dizem respeito à Pedagogia Social e as bases legais que apontam a atuação do pedagogo em espaços não escolares como elemento crucial da Pedagogia nesse momento histórico em que são requeridas práticas educativas em contextos diversos.

As múltiplas aprendizagens oportunizadas nesta ação extensionista foram sistematizadas na produção do livro PEDAGOGIA SOCIAL: UM HORIZONTE EDUCATIVO PARA CONTEXTOS DIVERSOS que registrou as experiências e estudos. Este livro foi uma expressão concreta da seriedade com a qual o Programa foi desenvolvido.

A Pedagogia Social nos dias atuais vem se construindo como um espaço relevante e significativo de reconstrução da dignidade da pessoa humana. O que justificou a relevância deste Programa de extensão Pedagogia Social: práticas educativas em contextos diversos.

É possível assegurar que houve de fato desenvolvimento cognitivo, motor, emocional, afetivo, ético, espiritual com as crianças e adolescentes atendidas pelo Programa. As muitas atividades, oficinas, desenvolvidas com as crianças, o interesse, envolvimento, participação e, sobretudo, a realização das atividades propostas são a expressão concreta o desenvolvimento proporcionado ao público atendido; (05 projetos eram com crianças e adolescentes)

Os (as) extensionistas demonstraram muito interesse e dedicação do início ao fim do Programa, apresentando uma desenvoltura pedagógica nos diversos trabalhos pedagógicos realizados com as pessoas atendidas pelo Programa. Além disso, demonstraram aperfeiçoamento profissional e competência acadêmica nas apresentações realizadas nos seguintes eventos IV Congresso Nacional de Educação (CONEDU); Encontro Nacional de Extensão; X Fórum Internacional de Pedagogia (FIPED), VI SEMANA DE PEDAGOGIA do Centro de Formação de Professores. Também demonstraram competência acadêmica na produção de artigos que ajudaram a compor o livro Pedagogia Social: um horizonte educativo para contextos diversos.

Durante a Semana de Pedagogia, realizada no CFP, no período de 01 a 04 de outubro de 2018, tivemos um Encontro Especial, de toda a equipe do Programa com o Professor Dr. Constantin Xypas, que é especialista na Sociologia do 
improvável. Tal encontro foi para nós muito relevante, pois apontou estratégias de aprimoramento de ações futuras e, também, nos orientou formas de melhor sistematizar as ações desenvolvidas.

A realização deste Programa de Extensão em Pedagogia Social deixa-nos a sensação de que nossa responsabilidade social não pode ficar apenas na esfera do discurso. Reconhecemos que o alcance da ação extensionista é limita, considerando a demanda social que é imensa, entretanto a sua contribuição social é inegável.

A promoção do desenvolvimento humano nas casas de acolhimento de crianças e adolescentes sob medida protetiva, para quem só a presença já é tudo, encheu-nos de satisfação em saber que oferecemos um pouco de alento e profissionalidade para algumas pessoas humanas que vê em nós, não apenas um professor, mas alegria, esperança, consolo, dignidade. Felizmente, a extensão universitária, é uma forma concreta da universidade demonstrar compromisso social com os problemas que estão no seu entorno.

Não restam dúvidas de que saímos dessa experiência mais humanizados. E mais competentes também. Estudamos, pesquisamos, partilhamos saberes e produzimos academicamente.

A experiência vivenciada na Pedagogia Social efetivada por meio da extensão universitária também nos dá a certeza de que a extensão é uma extraordinária maneira de contribuir com a formação dos graduandos, oportunizando-os a se descobrirem como profissionais da educação que fazem a diferença.

Enfim, é possível assegurar que trabalhos dessa natureza consolidam a missão institucional da universidade de melhorar as condições de vida das pessoas nos espaços nos quais se situam.

\section{REFERÊNCIAS}

BRASIL. Conselho Nacional de Educação. Resolução CNE/CP n. 1, de 15 de maio de 2006. Institui as Diretrizes Curriculares Nacionais para o Curso de Graduação em Pedagogia, licenciatura. Brasília: Conselho Nacional de Educação, 2006. Diário Oficial da União, ano CXLIII, n. 92, Brasília-DF, 16 maio de 2006a. Seção 1, p. 11. Disponível em:<http:// portal.mec.gov.br/cne/arquivos/pdf/rcp01_06.pdf>. Acesso em: 31/03/2018.

. Plano Nacional de Educação 2014-2024. Lei nº 13.005, de 25 de junho de 2014, que aprova o Plano Nacional de Educação (PNE) e dá outras providências. - Brasília: Câma $\neg$ ra dos Deputados, Edições Câmara, 2014. Disponível em: <http://www. 
observatoriodopne.org.br/uploads/referen $\neg$ ce/file/439/documento-referencia.pdf> Acesso em: 05 de março de 2018.

Plano Nacional de Extensão Universitária - PNExt, 1996. Disponível em: $<$ http:// pdi.ufabc.edu.br/wp-content/ uploads/2011/09/Plano-Nacional-de-Extens\%C3\%A3oUᄀniversit\%C3\%A1ria-2011-2020.pdf> Acesso em 05 de março de 2018

BULGRAEN, Vanessa C. O papel do professor e sua mediação nos processos de elaboração do conhecimento. Disponível em: < http://www.conteudo.org.br/index.php/ conteudo/article/viewFile/46/39>. Acesso em: 10 de maio de 2019

CALIMAN, Geraldo Pedagogia Social: seu potencial crítico e transformador. Disponível em: $\quad<$ https://sites.unicentro.br/wp/cursodepedagogia/files/2011/08/caliman-pedagogia-social-transformadora.pdf> Acesso em: 10 de maio de 2019

FREIRE, Paulo. Pedagogia da autonomia: saberes necessários à prática educativa. $55^{\mathrm{a}} \mathrm{ed}$. São Paulo: Paz e Terra, 2017.

Pedagogia do Oprimido - 64a . Ed. - São Paulo: Paz e Terra, 2017.

GRACIANI, Maria Stela Santos. Pedagogia social. São Paulo: Cortez, 2014.

SANTOS, Fabiana Ferreira dos. A relação existente entre o desenvolvimento motor e a aprendizagem em crianças de 2 a 7 anos. 2005. 47f. Trabalho de Conclusão de Curso (Pós-Graduação). Universidade Cândido Mendes, Rio de Janeiro, 2005. Disponível em: $<$ http://www.avm.edu.br/monopdf/6/FABIANA\%20FERREIRA\%20DOS\%20SANTOS.pdf $>$. Acesso em: 30 de março de 2018.

SEVERO, José Leonardo Rolim de Lima. O horizonte da Pedagogia Social: perspectiva de aproximação conceitual. Revista Ibero-Americana de Estudos em Educação, Araraquara, v. 12, n. 4, p. 2122-2137, out./dez. 2017. Disponível em: . E-ISSN: 1982-5587.

SEVERO, José Leonardo Rolim de Lima; MACHADO, Érico Ribas; RODRIGUES, Marli de Fátima. Pedagogia, pedagogia social e educação social no Brasil: entrecruzamentos, tensões e possibilidades. Interfaces científicas - educação. Aracaju, v.3, n.1, 2015, p.11-20. 